This item was submitted to Loughborough's Research Repository by the author.

Items in Figshare are protected by copyright, with all rights reserved, unless otherwise indicated.

\title{
Self-organizing TDMA MAC protocol for effective capacity improvement in IEEE 802.11 WLANs
}

PLEASE CITE THE PUBLISHED VERSION

http://dx.doi.org/10.1109/GLOCOMW.2015.7414195

PUBLISHER

(C) IEEE

VERSION

AM (Accepted Manuscript)

LICENCE

CC BY-NC-ND 4.0

\section{REPOSITORY RECORD}

Khan, Yahya, Mahsa Derakhshani, Saeedeh Parsaeefard, and Tho Le-Ngoc. 2019. "Self-organizing TDMA MAC Protocol for Effective Capacity Improvement in IEEE 802.11 Wlans". figshare.

https://hdl.handle.net/2134/21331. 


\title{
Self-Organizing TDMA MAC Protocol for Effective Capacity Improvement in IEEE 802.11 WLANs
}

\author{
Yahya Khan*, Mahsa Derakhshani ${ }^{\dagger}$, Saeedeh Parsaeefard*, Tho Le-Ngoc* \\ *Department of Electrical \& Computer Engineering, McGill University, Montreal, QC, Canada \\ ${ }^{\dagger}$ Department of Electrical \& Computer Engineering, University of Toronto, Toronto, ON, Canada \\ Email: yahya.khan@mcgill.ca; mahsa.derakhshani@utoronto.ca; saeideh.parsaeifard@mcgill.ca; tho.le-ngoc@mcgill.ca
}

\begin{abstract}
This paper presents a MAC protocol named selforganizing time division multiple-access (SO-TDMA) aiming to enable quality-of-service $(\mathrm{QoS})$ provisioning for delay-sensitive applications. Channel access operation in SO-TDMA is similar to carrier-sense multiple-access (CSMA) in the beginning, but quickly converges to TDMA with an adaptive pseudo-frame structure. This approach has the benefits of TDMA in a highload traffic environment, while overcoming its disadvantages in low-load, heterogeneous traffic scenarios. Furthermore, it supports distributed and asynchronous channel-access operation as in CSMA. These are achieved by dynamically adapting the transmission opportunity duration based on the common idle/busy channel state information acquired by each node through learning, without explicit message passing. Performance comparison of CSMA, TDMA, and SO-TDMA in terms of effective capacity, system throughput, and collision probability is investigated.
\end{abstract}

\section{INTRODUCTION}

The IEEE 802.11 wireless local area networks (WLANs) are being widely deployed inside homes and offices as well as public hotspots. Its success owed much to its robust and flexible medium access control (MAC) protocol. The fundamental MAC mechanism of IEEE 802.11, called distributed coordination function (DCF), is a contention-based access scheme based on carrier-sense multiple-access with collision avoidance (CSMA/CA). Traditionally designed to handle only data traffic, DCF fails to provide guaranteed QoS for multimedia services with delay constraints. This problem mostly stems from inevitable collisions occurring in CSMA/CA [1], [2]. Specifically, as the traffic load increases, the number of collisions and hence the delay significantly increase due to the heavy contention among nodes using DCF.

To support QoS provisioning for real-time multimedia applications, there is extensive research focusing on improving the MAC layer protocol [3], [4]. The proposed MAC enhancement approaches in the literature can be categorized into (i) centralized protocols and (ii) distributed protocols. Centralized MAC protocols (e.g., [5], [6]) provide information sharing among nodes that is used to control MAC activities through the access point (AP). Thus AP centrally controls channel-access to all connected nodes through signaling overhead messages. These centralized reservation-oriented mechanisms provide capacity and delay guarantees but induce some overhead reducing network capacity and scalability. On the other hand, distributed mechanisms for MAC enhancement [7], [8] aim to improve QoS while preserving the robustness and flexibility.

Among the distributed MAC protocols, the concept of pseudo time division multiple-access (PTDMA) has gained interest of the research community in recent years [9]-[12]. In PTDMA, each active node starts its transmission through random access as in CSMA but after a node successfully transmits once and receives acknowledgment, it switches to a pseudo periodic transmission similar to TDMA. In case of a collision, the node switches back to random access. Although periodic transmission can provide slot reservation guarantees, it has a drawback of being inflexible to heterogeneous traffic.

This paper investigates the statistical QoS performance through effective capacity (EC) of PTDMA in comparison with CSMA. EC is a QoS-aware metric that determines the maximum constant arrival rate supported by a network, while satisfying a target statistical delay requirement [13]. It is observed that PTDMA improves EC in saturated traffic scenarios compared with CSMA due to the reduced collision probability. However, with unsaturated traffic our study reveals CSMA has better EC performance. This is due to PTDMA frame structure: time-slot size and frame-length, not being adaptable to variations in $N_{\mathrm{a}}$ (nodes with packets in queue and sufficient channel gain). This results in channel underutilization in PTDMA leading to higher delays since parts of the channel are left unoccupied even though the active nodes have more packets to send. However, CSMA with an opportunistic behavior is flexible to traffic demand, thus having an advantage over PTDMA in unsaturated traffic.

In order to overcome this problem, we propose a cognitive Self-Organizing TDMA (SO-TDMA) MAC protocol. In this protocol, each node starts transmission using CSMA and then switches to periodic transmission as in PTDMA. But, different from PTDMA, the wireless channel frame structure in SOTDMA is adaptable to the changing traffic and channel conditions. Its fully distributed MAC algorithm enables each node to independently adapt its transmission length to maximize channel utilization based on locally available information. It is shown that SO-TDMA can provide QoS provisioning, improving EC as compared with CSMA and PTDMA in both saturated and unsaturated traffic scenarios.

The rest of this paper is organized as follows. Section II describes the system model for the MAC protocols along with the evaluated performance metrics. Section III presents the proposed SO-TDMA MAC protocol followed by simulation results in Section IV. Finally, Section V concludes the paper. 


\section{SySTEM MODEL}

\section{A. Network Model}

We consider a 802.11-based WLAN with a single AP and a set of nodes $\mathcal{N}=\{1, \cdots, N\}$. Each node (e.g., $n$ ) contends for the common channel with average arrival bit rate $\mu_{n}$. Suppose $Q_{n}$ denotes the queue length of node $n$, i.e., the number of backlogged packets in its queue. Accordingly, the number of active nodes (i.e., $N_{\mathrm{a}}$ ) is defined as the number of nodes with $Q_{n}>0$ whose instantaneous SNR is also above the minimum required threshold for transmission.

We assume a slotted transmission, where $T$ denotes the length of the smallest unit of time called a backoff timeslot. The limit of a single transmission opportunity of node $n$ is represented by $T_{\mathrm{s}, n}$ (in terms of backoff time-slots). The required number of backoff time-slots to transmit one packet is $S_{\text {req, } n}=P_{\mathrm{s}} /\left(R_{n} \times T\right)$, where $P_{\mathrm{s}}$ is the fixed packet size and $R_{n}$ is the transmission data rate of node $n$.

A block fading channel model is assumed, i.e. each node's channel gain remains constant for a time block determined by channel coherence time $T_{\mathrm{c}}$. It varies from one block to another independently and asynchronously. The transmission data rate of node $n$ (i.e., $R_{n}$ ) depends on the instantaneous channel power gain reported back to each node by the AP. Based on this information, a limited number of transmission modes (e.g., $K$ ) corresponding to certain transmission rates are selected that guarantee a minimum packet error rate (PER) for each node. Let $g_{n}$ be the channel power gain between the node $n$ and the AP, the instantaneous SNR is given as:

$$
S N R_{n}=P g_{n} / \sigma^{2}
$$

where $P$ is the transmit power of the node and $\sigma^{2}$ be the noise power. Each transmission mode (e.g., $k \in\{1, \ldots, K\}$ ) has a minimum SNR threshold $\eta_{k}$ to be satisfied in order to transmit at its corresponding channel rate $R_{k}$. Each time before transmission the channel rate $R_{k}$ selected for transmission of packets is the one that corresponds to the maximum $\eta_{k}$ that satisfies $S N R_{n} \geq \eta_{k}$. If $S N R_{n}$ is below $\eta_{1}$ (the lowest $\eta_{k}$ ) threshold, no transmission takes place.

\section{B. Medium Access Control}

In this paper, we intend to evaluate the QoS performance of existing MAC protocols. Learning from their strengths and weaknesses, we present a new MAC scheme, named SO-TDMA, that benefits from both CSMA and PTDMA. Implementation details of these protocols are presented below.

1) CSMA: This protocol requires nodes with packets to perform channel sensing for a period of time called distributed inter-frame space (DIFS). If the channel remains idle during this period, the node can transmit. Otherwise, if a transmission is detected before end of DIFS duration, it continues to wait until the channel is found idle for DIFS duration. Here it performs backoff (countdown) for a random time period chosen uniformly from the range $[0, w-1] . w$ is known as the contention window given in terms of backoff time-slots. Each node is initiated with $w$ equal to the minimum value of $C W_{\min }$. In case of a failed attempt, the value of $w$ is doubled up to the maximum contention window size $C W_{\max }$ [14].
If the channel is sensed busy during countdown, the node will freeze its backoff counter, resuming the countdown when channel is idle again. A node transmits when the backoff counter reaches zero. For each transmission opportunity, the node can transmit multiple back-to-back packets for a fixed period of time equal to $T_{\mathrm{s}, n}$. Once the packet is received successfully, the AP waits for a period of time called short inter-frame space (SIFS) and then sends an acknowledgment (ACK). If an ACK is not received by the node, $w$ is doubled and node attempts to retransmit the packet [14]. Upon successful transmission, $w$ is reset to $C W_{\min }$.

CSMA performs well for bursty heterogeneous traffic scenarios, however, suffers from performance degradation under heavy contention/traffic resulting from large number of collisions [1], [2]. Consequently, nodes experiencing collisions get starved as their contention window increases exponentially resulting in large packet delays and poor QoS. Therefore, CSMA-based MAC protocols are not able to guarantee QoS for delay sensitive multimedia applications. Consequently, a number solutions have been proposed incorporating TDMA concepts with the goal of overcoming these shortcomings.

2) PTDMA: One good example of such TDMA-based MAC protocols is pseudo-TDMA (PTDMA) as proposed in [9]-[12]. The basic concept of PTDMA is illustrated in Figure 1. Here initial channel-access for a node is the same as in CSMA using the exponential random backoff. However, after the first successful channel-access, a node with more packets in queue enters the TDMA phase where it employs a periodic backoff that is equal to the pseudo-frame length $T_{f}$. Subsequently, each successive transmission is repeated periodically assuming a reserved pseudo-slot of length $T_{\mathrm{s}, n}$ until all the packets in the queue have been sent. Carrier sensing is still performed before each transmission but without any additional backoff. A node switches to CSMA phase if the channel is found busy at the reserved pseudo-slot or in case of a collision (for example with a new incoming node trying to access the channel for the first time).

The important parameters of PTDMA: frame-length (i.e., $T_{f}$ ) and transmission time-slot size (i.e., $T_{\mathrm{s}, n}$ ) determine the performance of the protocol as they directly impact the channel utilization, throughput, and packet delay. If $T_{f}$ is too large, time between successive transmissions and thus packet delay becomes large. Otherwise, a value too small results in wastage of resources from unoccupied slots left out in each frame for new nodes to get access. Similarly, the transmission length $T_{\mathrm{s}, n}$ needs to be ideally updated as $T_{f} / N_{\mathrm{a}}$, otherwise there will be gaps left between transmissions again leading to wastage of resources. However, in reality, the instantaneous knowledge of $N_{\mathrm{a}}$ (based on the current queue length information and channel conditions of each node) is not available to nodes in the network. Therefore, $T_{\mathrm{s}, n}$ is set to a fixed value in PTDMA, e.g., $T_{\mathrm{s}, n}=T_{f} / N$ assuming that the value of the total number of nodes $N$ is known. In the rest of this paper, PTDMA with instantaneous information of $N_{\mathrm{a}}$ and $T_{\mathrm{s}, n}=T_{f} / N_{\mathrm{a}}$ is referred to as "Ideal-PTDMA" which represents an upper bound.

3) SO-TDMA: In the proposed protocol, the knowledge of $N_{\mathrm{a}}$ is learned independently by each node without information 


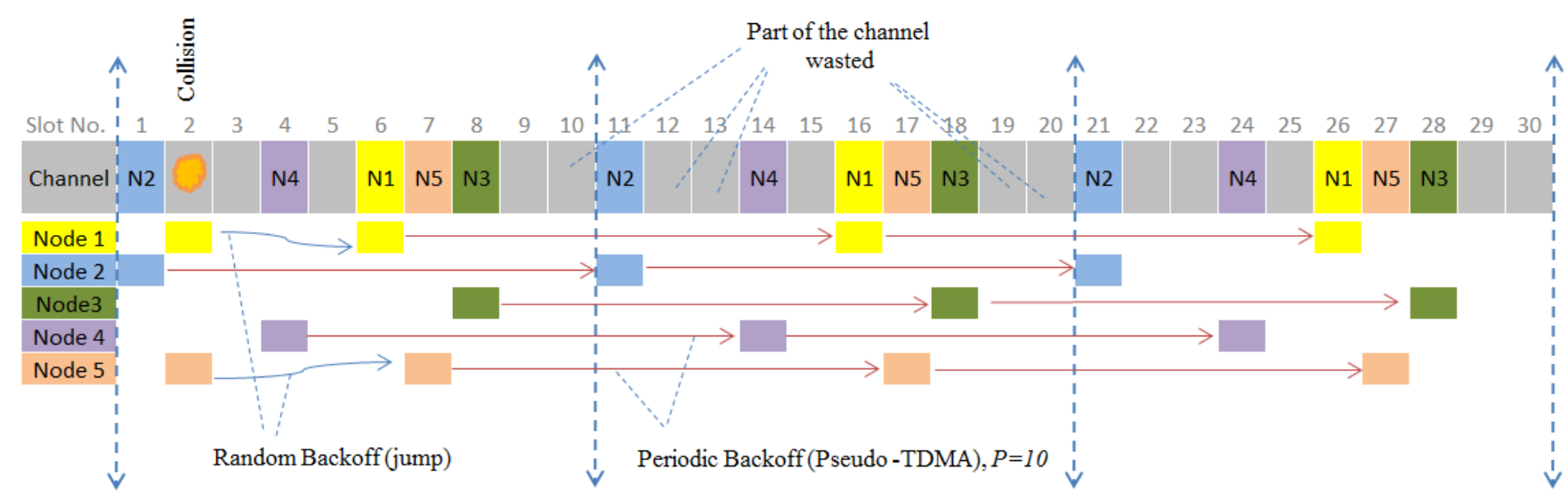

Fig. 1: Illustration of PTDMA

exchange among them. The key feature is the dynamically adaptive transmission length $T_{\mathrm{s}, n}$ in SO-TDMA defining its pseudo-frame structure that approaches the Ideal-PTDMA performance while frame-length $T_{f}$ is fixed. The proposed SO-TDMA algorithm is explained further in section III.

\section{Performance Metrics}

In order to assess and compare the performance of discussed MAC protocols, the following evaluation criteria are used.

1) System Throughput: To evaluate spectral efficiency of the protocols, we study system throughput $\left(S_{\text {sys }}\right)$ defined as

$$
S_{\text {sys }}=\sum_{n=1}^{N} S_{n}
$$

where $S_{n}$ is the throughput for node $n$ defined as the rate of successful packet transmission. Assuming a fixed packet size (i.e., $\left.P_{\mathrm{s}}\right), S_{n}$ can empirically be calculated as $\left(K_{\text {suc }, n} P_{\mathrm{s}}\right) / T_{\mathrm{m}}$ where $K_{\text {suc }, n}$ is the number of packets successfully transmitted by node $n$ and $T_{\mathrm{m}}$ is the measurement period.

2) Collision Probability: Since collisions are inevitable in random access schemes, the collision probability is an important measure of performance empirically calculated as

$$
P_{\mathrm{c}}=\frac{1}{N} \sum_{n=1}^{N} \frac{K_{\mathrm{col}, n}}{K_{\mathrm{tot}, n}},
$$

where $K_{\mathrm{col}, n}$ is the number of collided packets of node $n$ and $K_{\text {tot, } n}$ is the total number of packets transmitted by node $n$.

3) Effective Capacity: EC was first introduced in [13] as a QoS-aware metric that determines the maximum constant arrival rate supported by a network subject to a maximum tolerable delay-outage probability (i.e., probability that packet delay exceeds a target delay bound (e.g., $D_{\max }$ ). To empirically measure EC, an estimation procedure is proposed in [13], [15] where the delay-outage probability can be approximated as

$$
\operatorname{Pr}\left(D(t) \geq D_{\max }\right) \approx \hat{\gamma} e^{-\hat{\theta} D_{\max }},
$$

where $D(t)$ represents the total delay experienced by a packet, including queuing delay and negligible channel service delay at time-slot $t, \hat{\gamma}$ denotes the probability of non-empty queue, and $\hat{\theta}$ represents the delay-exponent. These parameters can be

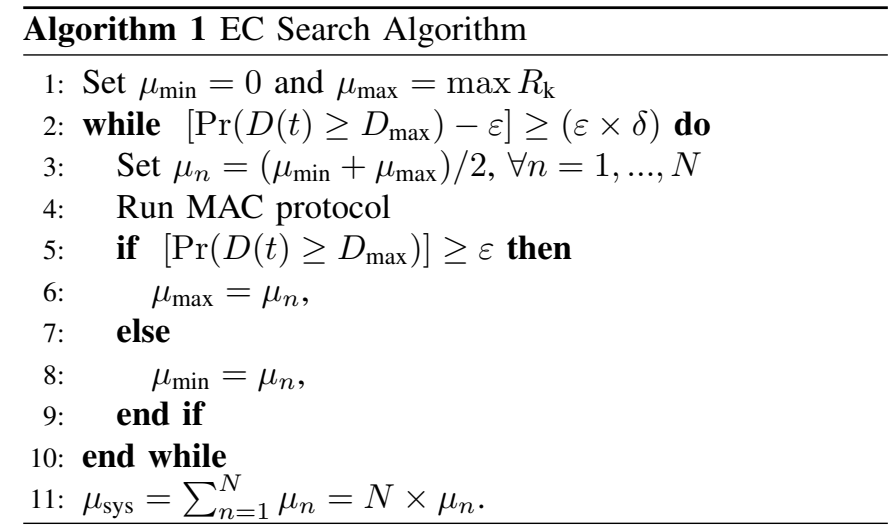

estimated by taking average over $N_{\mathrm{s}}$ samples collected during an interval of length $T_{\mathrm{m}}$ from all nodes as

$$
\hat{\gamma}=\frac{1}{N_{\mathrm{s}}} \sum_{k=1}^{N_{\mathrm{s}}} q_{k},
$$

and $\hat{\theta}=\hat{\gamma} / \hat{d}$ where $\hat{d}$ is the packet delay estimated by

$$
\hat{d}=\frac{1}{N_{\mathrm{s}}} \sum_{k=1}^{N_{\mathrm{s}}} D_{k} .
$$

More specifically, at the $k^{\text {th }}$ sampling epoch, the quantities of $q_{k} \in\{0,1\}$ (i.e., the indicator of whether a packet is in queue or not) and $D_{k}$ (i.e., the total delay experienced by a packet) are recorded. Given a fixed delay bound $D_{\max }$ in (4), supplying a traffic source with a constant rate $\mu$ shall result in a certain delay-outage probability. Thus, by iteratively testing different values of $\mu$, the value that is close enough to the target delay-outage probability threshold $\varepsilon$ can be found which is the desired EC. The bisection search method used to find EC is presented in Algorithm 1.

It is worth mentioning that we aim to measure EC for a 802.11 network and not a single wireless link. In Algorithm 1, all nodes are assumed to have homogeneous traffic load where each node's $\mu_{n}$ is identically varied to find the maximum value of $\mu_{\text {sys }}=N \times \mu_{n}$ that satisfies the statistical QoS constraint.

\section{Proposed SElf-Organizing TDMA (SO-TDMA)}

The proposed SO-TDMA protocol aims to improve the MAC layer channel utilization, throughput, and QoS in terms 
of packet delay. As discussed earlier in Section II-B, the key advantage of SO-TDMA over PTDMA is the ability to dynamically adapt the transmission time-slot of each node (i.e., $T_{\mathrm{s}, n}\left(f_{n}\right)$ ) to changing traffic and channel conditions, where $f_{n}$ represents the pseudo-frame index for node $n$. Thus, the goal with SO-TDMA is minimizing number of collisions as well as wastage of resources.

The access mechanism in SO-TDMA consists of two phases: 1) initial access phase where channel access is done via the random backoff procedure of CSMA; 2) periodic transmission phase where all active nodes independently attempt to fill the network pseudo-frame, maximizing channel utilization.

In the initial access phase (where $f_{n}$ is initially set to 0 ), nodes try to access the channel using CSMA with the transmission duration set to $T_{\mathrm{s}, n}\left(f_{n}\right)=T_{\mathrm{s}, n}(0)=T_{\text {start }}$. At the first successful channel access, a counter of length $T_{f}$ is initiated at the start of its transmission. The node stays in the initial access phase for all its subsequent transmissions until the counter expires (i.e., for one frame duration). In this phase, if the node empties its queue during any transmission (i.e., $\left.Q_{n}=0\right)$, the counter is reset to $T_{f}$. In case the counter expires and $Q_{n}>0$, the node would enter periodic transmission phase (Algorithm 2) until $Q_{n}$ becomes 0 again.

In the periodic transmission phase, all nodes periodically transmit with the same backoff value equal to $T_{f}$. At the time of each node's respective transmission, channel sensing is performed and starts transmission if the channel is idle for DIFS. The time-slot size for next frame $T_{\mathrm{s}, n}\left(f_{n}+1\right)$ is updated according to Algorithm 2. In case the channel is found busy, the node returns to the initial access phase.

In Algorithm 2, $T_{\min }$ and $T_{\max }$ are considered as the lower and upper bounds on the value of $T_{\mathrm{s}, n}\left(f_{n}\right)$ respectively. $T_{\min }$ is set to an arbitrary value so that the payload is still a significant portion of a transmission as compared to the overheads. Moreover, we need to have $T_{\max } \leq T_{f}-I_{\text {th }}$ in order to ensure at least $I_{\text {th }}$ idle time-slots in any frame. $I_{\text {th }}$ is the target number of idle time-slots per frame to ensure non-active nodes have an opportunity for random access.

In order to acquire an estimation of the network load and the number of active nodes $N_{\mathrm{a}}$, each node measures the number of idle time-slots during its pseudo-frame (between two consecutive periodic transmissions). Thus, in Algorithm 2 , each node keeps track of $I_{n}\left(f_{n}\right)$ or the number of idle slots sensed by node $n$ during the pseudo-frame $f_{n}$. Since the pseudo-frames of different nodes are asynchronous, their $I_{n}\left(f_{n}\right)$ measurements are expected to be different. However, assuming all nodes can perfectly sense the channel status (idle or busy), a moving average $\bar{I}_{n}\left(f_{n}\right)$ over subsequent pseudoframes for all active nodes converges to the same value.

$$
\bar{I}_{n}\left(f_{n}\right)=\alpha I_{n}\left(f_{n}\right)+(1-\alpha) \bar{I}_{n}\left(f_{n}-1\right),
$$

where $0<\alpha<1$.

Based on the value of $\bar{I}_{n}\left(f_{n}\right)$, each node calculates $I_{\text {gap }, n}\left(f_{n}\right)=\left|\bar{I}_{n}\left(f_{n}\right)-I_{\text {th }}\right|$. It tries to ensure at least $I_{\text {th }}$ idle time-slots in all pseudo-frames by making $I_{g a p, n}\left(f_{n}\right) \approx 0$, $\forall f_{n}$. To reach this goal, each node adaptively adjusts its transmission time-slot size for the next frame, i.e., $T_{\mathrm{s}, n}\left(f_{n}+1\right)$, based on the information from the last frame: $T_{\mathrm{s}, n}\left(f_{n}\right)$ and

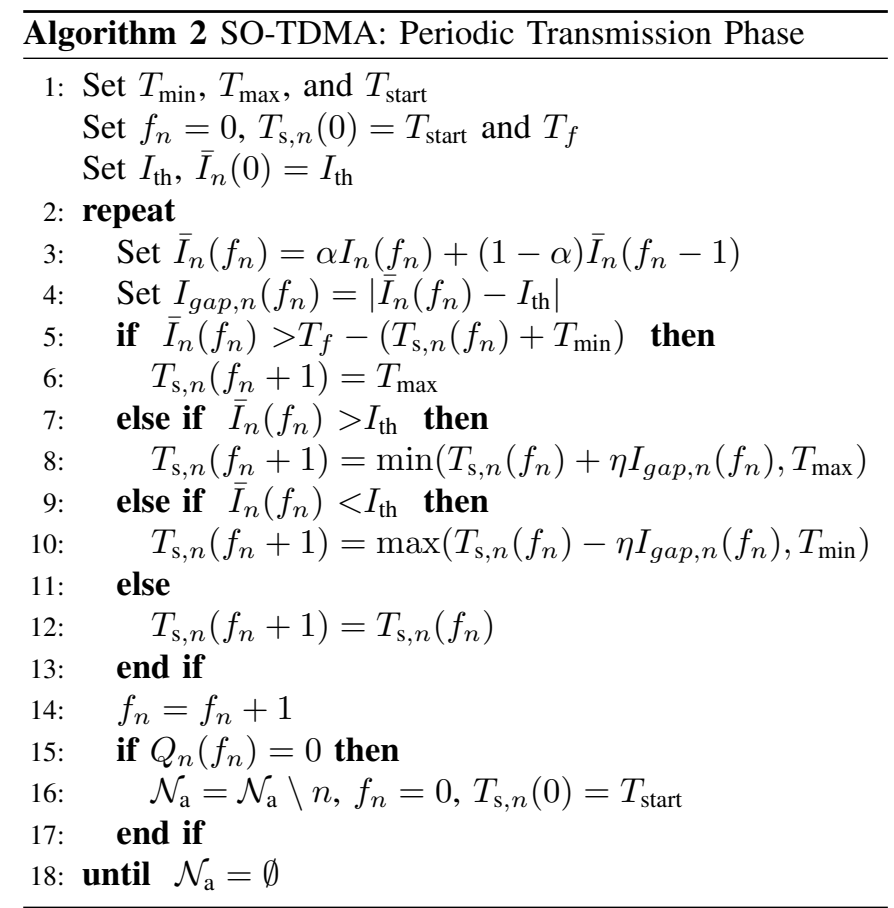

Channel Graph, N=10

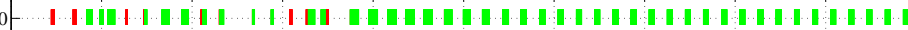

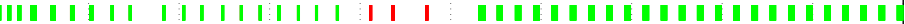

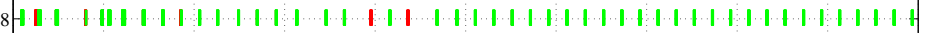

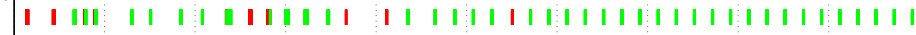

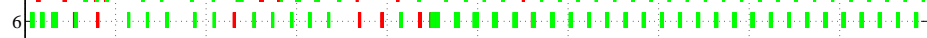
|||| ||| | | |

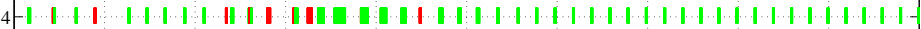

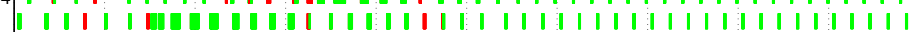

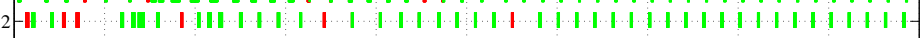

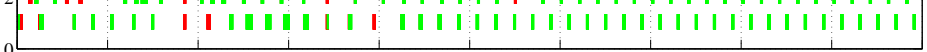

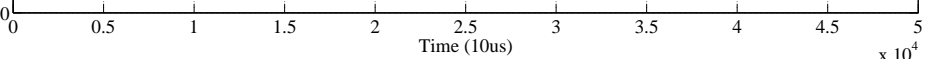

Fig. 2: SO-TDMA channel, $N=10$, based on Algorithm 2

$I_{\text {gap }, n}\left(f_{n}\right)$. This main functionality is described in Lines 5-13 of Algorithm 2 comprising three conditions. First condition in Line 5 describes the case that there is only one active node in the pseudo-frame $f_{n}$, i.e., $N_{\mathrm{a}}=1$. In this case, to reach the maximum utilization, in Line 6 , the active node sets $T_{\mathrm{s}, n}\left(f_{n}+1\right)=T_{\max }=T_{f}-I_{\mathrm{th}}$. Second condition in Line 7 checks if the frame is underutilized, i.e., $\bar{I}_{n}\left(f_{n}\right)>I_{\mathrm{th}}$. Therefore, $T_{\mathrm{s}, n}$ needs to be increased via the formulation of Line 8 where the amount of increase is relative to $I_{g a p, n}\left(f_{n}\right)$. This is defined here as $\eta I_{g a p, n}\left(f_{n}\right)$ where $\eta \in(0,1)$. Third condition checks if the frame is over-utilized through $\bar{I}_{n}\left(f_{n}\right)<I_{\text {th }}$. Consequently, $T_{\mathrm{s}, n}$ needs to be decreased via formulation of Line 10. This iterative process runs independently and in a distributed manner on node $n$ until $Q_{n}\left(f_{n}\right)=0$ (Line 15). As shown in Figure 2, after a few initial collisions (dark red), steady-state periodic transmission is achieved along with good channel utilization by adapting transmission lengths.

Remark 1: Comparison with PTDMA: The major difference in SO-TDMA compared with PTDMA is that the transmission length of each node, $T_{\mathrm{s}, n}$, is not fixed. Rather, it is distributively optimized during the periodic transmission phase in order to maximize channel utilization. The value of the transmission time-slot size $T_{\mathrm{s}, n}\left(f_{n}\right)$ in the case of PTDMA is fixed to $T_{\mathrm{s}, n}\left(f_{n}\right)=T_{f} / N$, while in SO-TDMA 


\begin{tabular}{|c|c|}
\hline Simulation Parameter & Value \\
\hline$T_{\mathrm{m}}$ & $50 \mathrm{~s}$ \\
\hline$N$ & $2-10$ \\
\hline$B$ & $20 \mathrm{MHz}$ \\
\hline$T$ & $10 \mu \mathrm{s}$ \\
\hline$I_{\text {th }}$ & $30 \times T$ \\
\hline$\overline{T_{f}}$ & $1000 \times T$ \\
\hline$T_{\min }$ & $40 \times T$ \\
\hline$T_{\max }$ & $T_{f}-I_{\mathrm{th}}=970 \times T$ \\
\hline$T_{\text {start }}$ & $100 T$ \\
\hline$T_{\mathrm{c}}$ & $10 \mathrm{~ms}$ \\
\hline$D_{\max }$ & $50 \mathrm{~ms}$ \\
\hline Average SNR & $20 \mathrm{~dB}$ \\
\hline DIFS & $4 \times T$ \\
\hline SIFS & $1 \times T$ \\
\hline$\overline{P \mathrm{~s}}$ & 2400 bytes \\
\hline $\mathrm{CW}_{\min }$ & 16 \\
\hline $\mathrm{CW}_{\max }$ & 1024 \\
\hline$\alpha$ & 0.7 \\
\hline$\varepsilon$ & 0.001 \\
\hline$\delta, \eta$ & 0.5 \\
\hline
\end{tabular}

TABLE I: Simulation Parameters

\begin{tabular}{|c|c|}
\hline$R_{\mathrm{k}}(\mathrm{Mbps})$ & SNR $(\mathrm{dB})\left[\eta_{k}, \eta_{k+1}\right)$ \\
\hline 6 & {$[5,8)$} \\
\hline 9 & {$[8,10)$} \\
\hline 12 & {$[10,13)$} \\
\hline 18 & {$[13,16)$} \\
\hline 24 & {$[16,19)$} \\
\hline 36 & {$[19,22)$} \\
\hline 48 & {$[22,25)$} \\
\hline 54 & {$[25, \infty)$} \\
\hline
\end{tabular}

TABLE II: Transmission rates $R_{\mathrm{k}}$ vs. SNR ranges used in the illustrative results

it changes from a starting value of $T_{\text {start }}$ to ensure maximum channel utilization in each pseudo-frame $f_{n}$. The number of active nodes, i.e., $N_{\mathrm{a}}(t)$, is a random function that depends on the traffic of nodes and their channel condition. SO-TDMA algorithm adapts according to this value, while PTDMA is inflexible to fluctuating heterogeneous traffic demand and random channel gains. Therefore, SO-TDMA allows nodes in the network to optimize the pseudo-frame structure according to the changing channel and traffic conditions, while approaching the performance of the Ideal-PTDMA. Since for the Ideal-PTDMA algorithm, the instantaneous value of $N_{\mathrm{a}}(t)$ is assumed to be known, its performance is optimal.

\section{ILlustrative Results}

In this section, the simulation setup is described, then followed by illustrative results to evaluate performance of SOTDMA in comparison with CSMA and PTDMA.

\section{A. Simulation Setup and Assumptions}

In this paper, we assume a Rayleigh fading channel as in [13], [16] where the channel power gain has an exponential distribution and is independent and identically distributed for

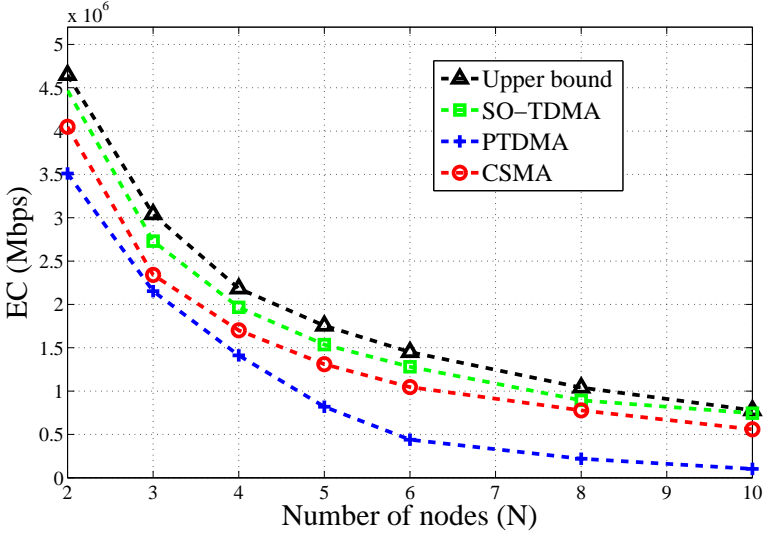

Fig. 3: Effective capacity vs. number of nodes

all nodes. The packet arrival for each node follows a Poisson process where $\mu_{n}$ is the mean arrival bit rate for node $n$. The parameter settings used for all MAC protocols are summarized in Tables I and II. The value of $T_{\mathrm{s}, n}$ is fixed to $T_{\mathrm{s}, n}=T_{\text {start }}$ for CSMA (in CSMA, TXOP or transmission opportunity limit for voice packets is $1.5 \mathrm{~ms}$ which is comparable to $T_{\mathrm{s}, n}$ value used here), $T_{\mathrm{s}, n}=T_{f} / N$ for PTDMA, and $T_{\mathrm{s}, n}=T_{f} / N_{\mathrm{a}}$ for Ideal-PTDMA. For SO-TDMA, the value of $T_{\mathrm{s}, n}$ is optimized as described in the previous section. The actual transmission length depends on the amount of data in buffer with $T_{\mathrm{s}, n}$ as the maximum transmission limit. Simulations for all protocols have been performed in MATLAB.

\section{B. Effective Capacity}

The comparison in terms of EC (normalized per number of nodes) is shown in Figure 3 plotted against the number of nodes $(N)$. The EC of SO-TDMA protocol approaches the upper bound (i.e., Ideal-PTDMA) and is significantly better than CSMA. Hence, in terms of QoS, SO-TDMA protocol shows better performance than CSMA for different values of $N$. It can be observed that the performance gain of SO-TDMA over CSMA is $10-30 \%$ in terms of effective capacity. It can be noted that higher percentage gain is achieved with increasing number of nodes (10\% with 2 nodes and $30 \%$ with 10 nodes) as in this case CSMA suffers from more collisions.

\section{Delay-outage Probability}

Figure 4 shows the delay-outage probability of all nodes in different protocols against the mean arrival rate. It is found to be the lowest for Ideal-PTDMA protocol for all values of $\mu_{\text {sys }}$ as expected. Interestingly, the proposed SOTDMA performance is very close to the lower bound and outperforms both CSMA and PTDMA. Thus, for a fixed acceptable threshold for delay-outage probability (as QoS requirements), much larger effective capacity can be supported in SO-TDMA than CSMA and PTDMA. For example, Figure 4 shows that to maintain a delay-outage probability of $10^{-3}$, SO-TDMA offers an effective capacity of $8 \mathrm{Mbps}$ or $95 \%$ of the lower-bound of $8.4 \mathrm{Mbps}$, while CSMA achieves $75 \%$ and PTDMA only $45 \%$.

\section{System Throughput}

To investigate the maximum achievable system throughput over the wireless channel without delay constraint, Figure 5 


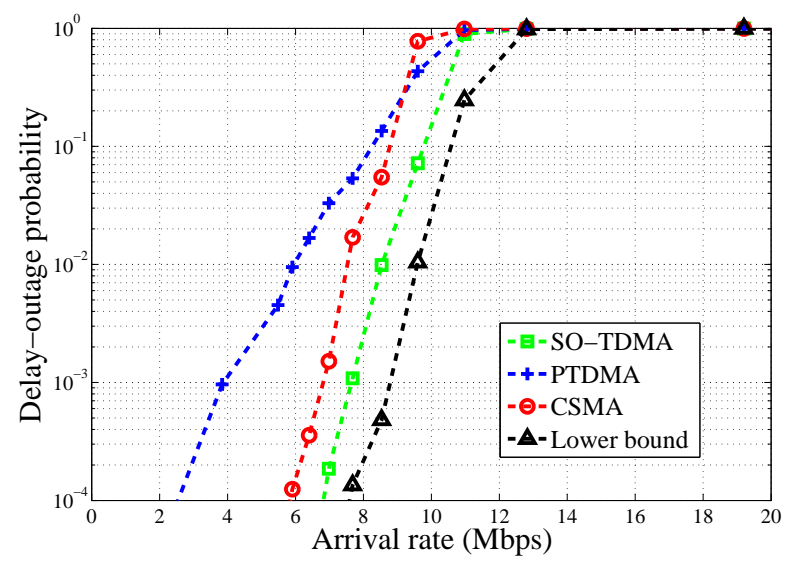

Fig. 4: Delay-outage probability vs. arrival rate $\mu_{\text {sys }}, N=5$

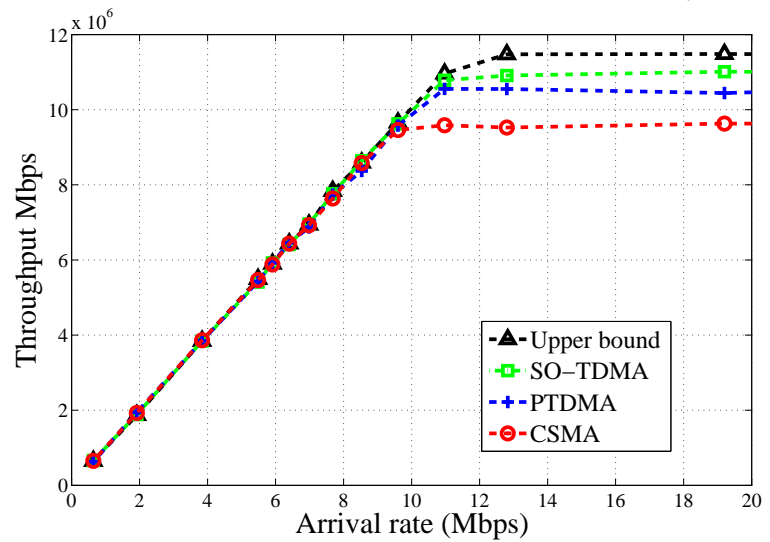

Fig. 5: System throughput vs arrival rate $\mu_{\text {sys }}, N=5$

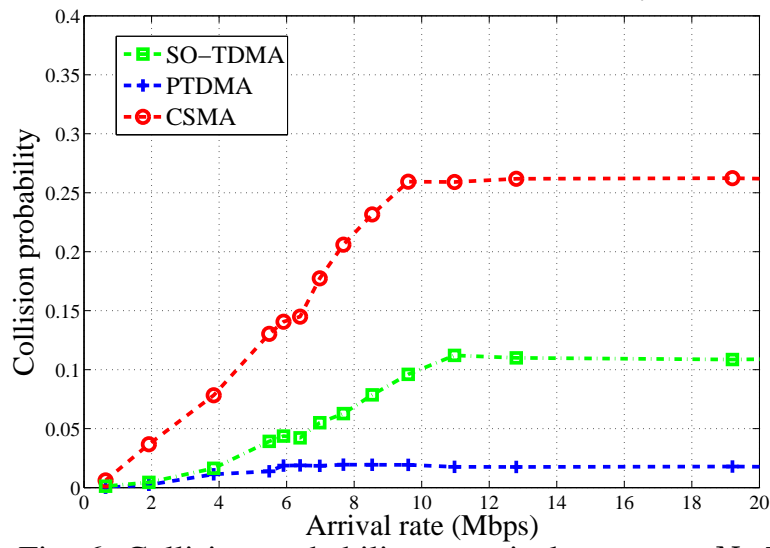

Fig. 6: Collision probability vs arrival rate $\mu_{\text {sys }}, N=5$

plots $S$ aggregated over all nodes as $\mu_{\text {sys }}$ is varied between 0 to $20 \mathrm{Mbps}$. When the load (arrival rate) is low the system throughput $S$ in all the protocols increases linearly with $S=$ $\mu_{\text {sys }}$ as expected. However, as the system load increases further in a higher range, the system throughput reaches saturation at 9.5 Mbps for CSMA, 10.5 Mbps for PTDMA, $11 \mathrm{Mbps}$ for SO-TDMA, and 11.5 Mbps in the case of upper-bound. The proposed SO-TDMA outperforms both PTDMA and CSMA.

\section{E. Collision Probability}

Collision probability for the protocols is shown in Figure 6. Collision probability in SO-TDMA is significantly less than CSMA but still greater than PTDMA. However, with the greater risk of collisions compared to PTDMA, much better performance in terms of EC has been achieved in SO-TDMA.

\section{CONCLUSION}

In this paper, using the concept of effective capacity, it is shown that the existing MAC protocols cannot guarantee the maximum tolerable delay for multimedia services in WLANs. To improve this aspect, we proposed a distributed and selforganizing protocol, SO-TDMA, that utilizes both CSMA and TDMA to form a dynamic, asynchronous pseudo-frame structure for transmission and access. Simulation results illustrate the effectiveness of the proposed SO-TDMA in improving throughput, channel utilization, and effective capacity. The proposed SO-TDMA can approach the performance in the ideal case (upper bound) which needs complete and precise information about queue lengths and channel conditions of all nodes. As a future study, we recommend the study of the impact on access delay and fairness among nodes in a multicell environment to further develop the scheme as a potential candidate for the upcoming IEEE 802.11ax standard.

\section{REFERENCES}

[1] S. Choi, K. Park, and C.-k. Kim, "On the performance characteristics of WLANs: Revisited," in Proc. ACM International Conf. Measurement and Modeling Comput. Syst. (SIGMETRICS), 2005, pp. 97-108.

[2] J. Robinson and T. Randhawa, "Saturation throughput analysis of IEEE 802.11e enhanced distributed coordination function," IEEE J. Sel. Areas Commun., vol. 22, no. 5, pp. 917-928, Jun. 2004.

[3] E. Charfi, L. Chaari, and L. Kamoun, "PHY/MAC enhancements and QoS mechanisms for very high throughput WLANs: A survey," IEEE Commun. Surveys Tuts., vol. 15, no. 4, pp. 1714-1735, Apr. 2013.

[4] M. Derakhshani and T. Le-Ngoc, "Cognitive MAC designs: Background," in Cognitive MAC Designs for OSA Networks. Springer International Publishing, 2014, pp. 15-31.

[5] W. Ye, J. Heidemann, and D. Estrin, "Medium access control with coordinated, adaptive sleeping for wireless sensor networks," IEEE/ACM Transactions on Networking, vol. 12, pp. 493-506, 2003.

[6] I. Rhee, A. Warrier, M. Aia, J. Min, and M. Sichitiu, "Z-MAC: A hybrid MAC for wireless sensor networks," Networking, IEEE/ACM Transactions on, vol. 16, no. 3, pp. 511-524, June 2008.

[7] K. Almotairi and X. Shen, "A distributed multi-channel MAC protocol for ad hoc wireless networks," Mobile Computing, IEEE Transactions on, vol. 14, no. 1, pp. 1-13, Jan 2015.

[8] L. T. Tan and L. B. Le, "Distributed MAC protocol for cognitive radio networks: Design, analysis, and optimization," Vehicular Technology, IEEE Transactions on, vol. 60, no. 8, pp. 3990-4003, Oct 2011.

[9] M. Lee, Y. Kim, and C.-H. Choi, "Period-controlled MAC for high performance in wireless networks," IEEE/ACM Trans. Netw., vol. 19, no. 4, pp. 1237-1250, Aug. 2011.

[10] G. Jakllari, M. Neufeld, and R. Ramanathan, "A framework for frameless TDMA using slot chains," in Proc. IEEE Int. Conf. Mobile Adhoc and Sensor Systems (MASS), Oct. 2012, pp. 56-64.

[11] I. Tinnirello and P. Gallo, "Supporting a Pseudo-TDMA access scheme in mesh wireless networks," in Wireless Access Flexibility. Springer Berlin Heidelberg, 2013, vol. 8072, pp. 80-92.

[12] G. S. Paschos, I. Papapanagiotou, S. A. Kotsopoulos, and G. K. Karagiannidis, "A new MAC protocol with Pseudo-TDMA behavior for supporting quality of service in 802.11 wireless LANs," EURASIP J. Wireless Commun. Netw., vol. 2006, no. 1, 2006.

[13] D. Wu and R. Negi, "Effective capacity: A wireless link model for support of quality of service," IEEE Trans. Wireless Commun., vol. 2, no. 4, pp. 630-643, Jul. 2003.

[14] G. Bianchi, "Performance analysis of the IEEE 802.11 distributed coordination function," IEEE J. Sel. Areas Commun., vol. 18, no. 3, pp. 535-547, Mar. 2000.

[15] A. Davy, B. Meskill, and J. Domingo-Pascual, "An empirical study of effective capacity throughputs in 802.11 wireless networks," in Proc. IEEE Global Commun. Conf. (GLOBECOM), Dec. 2012.

[16] K. Masood, M. Sohail, and A. Sheikh, "Analyzing IEEE 802.11 DCF for FU-FB Systems under Multipath Fading Channels Using TUA," in Vehicular Technology Conference (VTC Spring), 2013 IEEE 77th, June 2013, pp. 1-5. 